\title{
The mean square of the Riemann zeta-function in the critical strip II
}

by

Konji Matsumoto (Morioka) and Tom Meurman (Turku)

1. Introduction. Let $\zeta(s)$ be the Riemann zeta-function. Suppose that $T \geq 2$ and define

$$
E(T)=\int_{0}^{T}\left|\zeta\left(\frac{1}{2}+i t\right)\right|^{2} d t-T\left(\log \frac{T}{2 \pi}+2 \gamma-1\right)
$$

where $\gamma$ is Euler's constant. It is known (see e.g. [2]) that $E(T) \ll T^{\theta}$, where $\theta$ is slightly less than $1 / 3$, but the average order of $E(T)$ is $T^{1 / 4}$. More precisely, defining

$$
F(T)=\int_{2}^{T} E(t)^{2} d t-\frac{2}{3}(2 \pi)^{-1 / 2} \frac{\zeta(3 / 2)^{4}}{\zeta(3)} T^{3 / 2}
$$

Heath-Brown [1] proved that $F(T) \ll T^{5 / 4} \log ^{2} T$. Meurman [6] and Motohashi [8], [9] independently improved this to $F(T) \ll T \log ^{5} T$, and Preissmann [10] (and independently, Ivić [3]) obtained a further improvement by showing that $F(T) \ll T \log ^{4} T$.

It is our purpose to investigate the analogue of $F(T)$ in the critical strip. This work was initiated by Matsumoto [5], and his paper contains most of the background relevant for this paper. From now on we assume that $1 / 2<\sigma<3 / 4$. Define

$$
E_{\sigma}(T)=\int_{0}^{T}|\zeta(\sigma+i t)|^{2} d t-\zeta(2 \sigma) T-(2 \pi)^{2 \sigma-1} \frac{\zeta(2-2 \sigma)}{2-2 \sigma} T^{2-2 \sigma}
$$

and

$$
F_{\sigma}(T)=\int_{2}^{T} E_{\sigma}(t)^{2} d t-c_{0} T^{5 / 2-2 \sigma}
$$


where

$$
c_{0}=\frac{2}{5-4 \sigma}(2 \pi)^{2 \sigma-3 / 2} \frac{\zeta(3 / 2)^{2}}{\zeta(3)} \zeta(5 / 2-2 \sigma) \zeta(1 / 2+2 \sigma) .
$$

Theorem 3 of Matsumoto [5] asserts that $F_{\sigma}(T) \ll T^{7 / 4-\sigma} \log T$. We shall prove the following refinement:

Theorem. For $1 / 2<\sigma<3 / 4$ and $T \geq 2$ we have

$$
F_{\sigma}(T)=O(T)
$$

where the implied constant depends on $\sigma$.

A simple argument, based on (3.29) below, shows that

$$
\int_{2}^{T} E_{\sigma}(t) d t \sim-2 \pi \zeta(2 \sigma-1) T .
$$

Thus perhaps $F_{\sigma}(T) \sim 4 \pi^{2} \zeta(2 \sigma-1)^{2} T$. This would imply that the estimate in our theorem is essentially the best possible. This is one of the reasons why we do not confine ourselves to the weaker estimate $F_{\sigma}(T) \ll T \log T$, the proof of which would be simpler. Indeed, to drop the log-factor here, we need an idea which has not been used in the papers concerning $F(T)$.

Another simple argument shows that if $F_{\sigma}(T) \ll T^{\alpha}(\log T)^{\beta}$ then $E_{\sigma}(T)$ $\ll T^{\alpha / 3}(\log T)^{\beta / 3}+T^{3 / 4-\sigma}$. Our theorem thus implies that $E_{\sigma}(T) \ll T^{1 / 3}$. But this is weaker than the estimate $E_{\sigma}(T) \ll T^{1 /(1+4 \sigma)} \log ^{2} T$ of Matsumoto [5]. The result $E_{\sigma}(T)=\Omega\left(T^{3 / 4-\sigma}\right)$, given also by Matsumoto [5], is not sufficient to yield an $\Omega$-result for $F_{\sigma}(T)$. However, we can prove that $E_{\sigma}(T)=\Omega\left(T^{3 / 4-\sigma}(\log T)^{\sigma-1 / 4}\right)$. We shall show this in a subsequent paper. Therefore it follows that

$$
F_{\sigma}(T)=\Omega\left(T^{9 / 4-3 \sigma}(\log T)^{3 \sigma-3 / 4}\right) .
$$

Notation. The symbols $O, \ll, \gg, \asymp$ and $\Sigma^{\prime}$ have their usual meaning (see p. xiii of [2]), and the implied constants may depend on $\sigma$.

Acknowledgements. We wish to express our gratitude to Prof. Aleksandar Ivić for his useful comments concerning this work.

2. Estimates involving $\sigma_{1-2 \sigma}(n)$. Define $\sigma_{a}(n)=\sum_{d \mid n} d^{a}$, as usual. We have the elementary estimates

$$
\sum_{n \leq x} \sigma_{1-2 \sigma}(n) \ll x, \quad \sum_{n \leq x} \sigma_{1-2 \sigma}(n)^{2} \ll x
$$

and

$$
\sum_{x<n \leq x+y} \sigma_{1-2 \sigma}(n) \ll y+\sqrt{x}
$$


In fact, the first estimate is obvious. Since

$$
\begin{aligned}
\sum_{n \leq x} \sigma_{1-2 \sigma}(n)^{2} & =\sum_{k m \leq x} \sigma_{1-2 \sigma}(k m) k^{1-2 \sigma} \\
& \leq \sum_{k m \leq x} \sigma_{1-2 \sigma}(m) \sigma_{1-2 \sigma}(k) k^{1-2 \sigma} \\
& \ll \sum_{m \leq x} \sigma_{1-2 \sigma}(m)(x / m)^{2-2 \sigma} \ll x,
\end{aligned}
$$

the second estimate follows. Since

$$
\begin{aligned}
& \sum_{x<n \leq x+y} \sigma_{1-2 \sigma}(n) \leq 2 \sum_{x<n \leq x+y} \sum_{\substack{d \mid n \\
d \leq \sqrt{n}}} d^{1-2 \sigma} \ll \sum_{d \leq \sqrt{x+y}} d^{1-2 \sigma} \sum_{\substack{x<n \leq x+y \\
n \equiv 0(\bmod d)}} 1 \\
& \ll \sum_{d \leq \sqrt{x+y}} d^{1-2 \sigma}(y / d+O(1)) \\
& \ll y+(x+y)^{1-\sigma} \ll y+\sqrt{x},
\end{aligned}
$$

the third estimate follows. The first two of these will be used several times without referring to them explicitly.

We have

$$
\sum_{n \leq x}^{\prime} \sigma_{1-2 \sigma}(n)=\zeta(2 \sigma) x+\frac{\zeta(2-2 \sigma)}{2-2 \sigma} x^{2-2 \sigma}-\frac{1}{2} \zeta(2 \sigma-1)+\Delta_{1-2 \sigma}(x),
$$

where

$$
\begin{aligned}
\Delta_{1-2 \sigma}(x)= & \frac{1}{\pi \sqrt{2}} x^{3 / 4-\sigma} \sum_{n=1}^{\infty} \sigma_{1-2 \sigma}(n) n^{\sigma-5 / 4} \\
& \times\left\{\cos (4 \pi \sqrt{n x}-\pi / 4)+\frac{c_{1}}{\sqrt{n x}} \sin (4 \pi \sqrt{n x}-\pi / 4)\right\} \\
& +O\left(x^{-1 / 4-\sigma}\right),
\end{aligned}
$$

$c_{1}$ depends only on $\sigma$ and the series is boundedly convergent for $x$ in any fixed finite interval (see Section 2 in [5]).

For any fixed $\varepsilon>0$ we have

$$
\int_{1}^{x} \Delta_{1-2 \sigma}(\xi)^{2} d \xi=c_{2} x^{5 / 2-2 \sigma}+O\left(x^{7 / 4-\sigma+\varepsilon}\right),
$$

where $c_{2}$ depends only on $\sigma$. This is a special case of Theorem 2 of Kiuchi [4].

3. A formula for $E_{\sigma}(T)$. The aim of this section is to obtain for $E_{\sigma}(T)$ a formula which is a refinement of Theorem 2 in [5] and, up to a minor modification, an analogue of Lemma 2 in [6]. 
By a straightforward calculation based on Stirling's formula

$$
\Gamma(z)=\sqrt{2 \pi} e^{-z} z^{z-1 / 2}\left(1+\frac{1}{12 z}+O\left(|z|^{-2}\right)\right)
$$

it can be shown that

$$
\operatorname{Re}\left(\frac{\Gamma(1-\sigma-i t)}{\Gamma(\sigma-i t)}\right)-\sin (\pi \sigma) t^{1-2 \sigma} \ll t^{-1-2 \sigma}
$$

for $t \geq 2$. Hence

$$
\int_{T}^{\infty}\left(\operatorname{Re}\left(\frac{\Gamma(1-\sigma-i t)}{\Gamma(\sigma-i t)}\right)-\sin (\pi \sigma) t^{1-2 \sigma}\right) d t \ll T^{-2 \sigma} .
$$

Denote the integral on the left by $c_{3}$ when $T=0$. It follows that

$$
\begin{aligned}
& \int_{0}^{T}\left\{\frac{\Gamma(1-\sigma-i t)}{\Gamma(\sigma-i t)}+\frac{\Gamma(1-\sigma+i t)}{\Gamma(\sigma+i t)}\right\} d t \\
& \qquad \frac{\sin (\pi \sigma)}{1-\sigma} T^{2-2 \sigma}+2 c_{3}+O\left(T^{-2 \sigma}\right) .
\end{aligned}
$$

We need not know the value of the constant $c_{3}$ to prove our theorem. But $c_{3}$ contributes to the constant term in (3.29) and therefore it may be of some interest to know that $c_{3}=0$. We prove this in the Appendix. Combining (3.1), where $c_{3}=0$, with formula (3.4) in [5], observing that

$$
\zeta(2 \sigma-1) \Gamma(2 \sigma-1) \sin (\pi \sigma)=\frac{1}{2}(2 \pi)^{2 \sigma-1} \zeta(2-2 \sigma)
$$

and recalling our definition of $E_{\sigma}(T)$ we obtain

$$
E_{\sigma}(T)=-i G_{\sigma}(T)+O\left(T^{-2 \sigma}\right)
$$

where

$$
G_{\sigma}(T)=\int_{\sigma-i T}^{\sigma+i T} g(z, 2 \sigma-z) d z
$$

and $g(z, 2 \sigma-z)$ is defined as at the beginning of Section 4 in [5].

Define

$$
f(T, x)=2 T \operatorname{arsinh} \sqrt{\frac{\pi x}{2 T}}+2 \pi x\left(\frac{T}{2 \pi x}+\frac{1}{4}\right)^{1 / 2}-\frac{\pi}{4}
$$

and

$$
q(x)=\left(\operatorname{arsinh} \sqrt{\frac{\pi x}{2 T}}\right)^{-1}\left(\frac{T}{2 \pi x}+\frac{1}{4}\right)^{-1 / 4}\left(\left(\frac{T}{2 \pi x}+\frac{1}{4}\right)^{1 / 2}+\frac{1}{2}\right)^{-1} .
$$

According to Sections 4 and 5 in [5] we have

$$
G_{\sigma}(T)=G_{1}-G_{2}+G_{3}-G_{4},
$$


where

$$
\begin{aligned}
G_{1}= & \frac{i}{\sqrt{2}}\left(\frac{T}{2 \pi}\right)^{1 / 2-\sigma} \sum_{n<X}(-1)^{n} n^{\sigma-1} \sigma_{1-2 \sigma}(n) \\
& \times\left(\operatorname{arsinh} \sqrt{\frac{\pi n}{2 T}}\right)^{-1}\left(\frac{T}{2 \pi n}+\frac{1}{4}\right)^{-1 / 4} \cos (f(T, n)) \\
& +O\left(T^{1 / 4-\sigma}\right),
\end{aligned}
$$

$$
\begin{aligned}
G_{2} & \ll T^{-1 / 2}\left|\Delta_{1-2 \sigma}(X)\right|, \\
G_{3} & =-2 \pi i \zeta(2 \sigma-1)+O\left(T^{\sigma-1}\right),
\end{aligned}
$$

$$
G_{4}=G_{40}+O\left(T^{1 / 2-\sigma} \int_{X}^{\infty}\left|\Delta_{1-2 \sigma}(\xi)\right| \xi^{\sigma-2} d \xi\right)
$$

$$
G_{40}=i \pi \sqrt{2}\left(\frac{T}{2 \pi}\right)^{3 / 2-\sigma} \int_{X}^{\infty} \Delta_{1-2 \sigma}(\xi) \xi^{\sigma-2} q(\xi) \cos (f(T, \xi)-\pi \xi+\pi / 2) d \xi
$$

$X=N+1 / 2 \asymp T$ and $N$ is an integer. In fact, the estimate (3.6) has not been displayed in [5], but it is implicitly there. The only reason for assuming $X=N+1 / 2$ was to ensure $X$ is not an integer. Hence the decomposition (3.4) remains valid even on the weaker assumption that $X$ is not an integer.

By Schwarz's inequality and (2.3) the integral in (3.7) is $O\left(X^{-1 / 4}\right)$ so that

$$
G_{4}=G_{40}+O\left(T^{1 / 4-\sigma}\right) .
$$

If $a \asymp T^{1 / 2}$ and $U \asymp T^{1 / 4}$ then the numbers $X=(a+u)^{2}$, where $0 \leq u \leq U$, satisfy $X \asymp T$, as required. Then, setting

$$
G_{j}^{*}=U^{-1} \int_{0}^{U} G_{j} d u,
$$

we deduce from (3.4) that

$$
G_{\sigma}(T)=G_{1}^{*}-G_{2}^{*}+G_{3}^{*}-G_{4}^{*},
$$

since $G_{\sigma}(T)$ is independent of $X$.

By Schwarz's inequality and (2.3) we have

$$
\begin{aligned}
G_{2}^{*} & \ll T^{-1 / 2} U^{-1} \int_{0}^{U}\left|\Delta_{1-2 \sigma}\left((a+u)^{2}\right)\right| d u \\
& \ll T^{-1 / 2} U^{-1} a^{-1} \int_{a^{2}}^{(a+U)^{2}}\left|\Delta_{1-2 \sigma}(x)\right| d x
\end{aligned}
$$




$$
\ll(T U a)^{-1 / 2}\left(\int_{a^{2}}^{(a+U)^{2}} \Delta_{1-2 \sigma}(x)^{2} d x\right)^{1 / 2} \ll T^{1 / 4-\sigma}+T^{-\sigma / 2+\varepsilon} .
$$

Trivially

$$
G_{3}^{*}=-2 \pi i \zeta(2 \sigma-1)+O\left(T^{\sigma-1}\right) .
$$

To evaluate $G_{4}^{*}$ consider $U^{-1} \int_{0}^{U} G_{40} d u$. Substitute the expression in (2.2) into the expression defining $G_{40}$. Then change the order of $\iint$ and $\sum$ and substitute $\xi=x^{2}$. This yields

$$
\begin{aligned}
U^{-1} \int_{0}^{U} G_{40} d u & \\
& =2 i\left(\frac{T}{2 \pi}\right)^{3 / 2-\sigma} \sum_{n=1}^{\infty} \sigma_{1-2 \sigma}(n) n^{\sigma-5 / 4} K_{n}+O\left(T^{1 / 4-\sigma}\right),
\end{aligned}
$$

where the $O$-term is due to the $O$-term in $(2.2)$ (note that $q(\xi) \ll 1$ for $\xi \geq X)$ and

$$
\begin{aligned}
K_{n}= & U^{-1} \int_{0}^{U} \int_{a+u}^{\infty} x^{-3 / 2} q\left(x^{2}\right) \cos \left(f\left(T, x^{2}\right)-\pi x^{2}+\pi / 2\right) \\
& \times\left\{\cos (4 \pi x \sqrt{n}-\pi / 4)+c_{1} n^{-1 / 2} x^{-1} \sin (4 \pi x \sqrt{n}-\pi / 4)\right\} d x d u .
\end{aligned}
$$

The term-by-term integration can be justified as in [5]. But here we show a different argument. The idea was suggested by Prof. Yoichi Motohashi. Clearly it is sufficient to consider only the "worse" part of the series in (2.2). Then, since this is boundedly convergent on finite intervals, it is sufficient to show that

$$
\lim _{\lambda \rightarrow \infty} \sum_{n=1}^{\infty} \sigma_{1-2 \sigma}(n) n^{\sigma-5 / 4} \int_{\lambda}^{\infty} x^{-3 / 2} q\left(x^{2}\right) e^{i h(x)} d x=0,
$$

where we have set $h(x)=f\left(T, x^{2}\right)-\pi x^{2} \pm 4 \pi x \sqrt{n}$. Since

$$
h^{\prime}(x)=2 T\left(\frac{x}{2}+\left(\frac{x^{2}}{4}+\frac{T}{2 \pi}\right)^{1 / 2}\right)^{-1} \pm 4 \pi \sqrt{n},
$$

it follows that $h^{\prime}$ is monotonic and $\left|h^{\prime}(x)\right| \geq \sqrt{n}$. Also the function $x^{-3 / 2} q\left(x^{2}\right)$ is monotonic and its value is $\ll x^{-3 / 2}$. It follows that the integral in our claim is $\ll \lambda^{-3 / 2} n^{-1 / 2}$ (using formula (2.3) in [2]), which proves our claim.

Consider $K_{n}$. Define

$$
\xi(T, n, a)=\max \left(0, \min \left(1, U^{-1}\left(\frac{T}{2 \pi \sqrt{n}}-\sqrt{n}-a\right)\right)\right)
$$


and

$$
B(y)=B(y, T)=\frac{T}{2 \pi}+\frac{1}{2} y^{2}-y\left(\frac{T}{2 \pi}+\frac{1}{4} y^{2}\right)^{1 / 2}
$$

so that $B(a+u)$ equals what was denoted by $Z(u)$ in [6]. For $y \geq 0$ we have

$$
n \leq B(y, T) \Leftrightarrow \frac{T}{2 \pi \sqrt{n}}-\sqrt{n} \geq y .
$$

Up to the value of $c_{1}$, which is irrelevant, the expression in (3.14) is exactly the same as $K_{n}$ in [6]. By Lemma 1 in [6] and after some simplifications depending on the fact that if $\xi(T, n, a) \neq 0$ then $n\langle B(a)$ and $T /(2 \pi)-n \gg$ $T$ (see (3.15) and (3.17)) we find that

$$
\begin{aligned}
K_{n}= & 2 \pi \xi(T, n, a) T^{-1} n^{1 / 4}\left(\log \frac{T}{2 \pi n}\right)^{-1} \cos \left(T \log \frac{T}{2 \pi n}-T+\frac{\pi}{4}\right) \\
& +O\left(U^{-1} T^{-3 / 4} \sum_{j=0}^{1} \min \left(1,(\sqrt{n}-\sqrt{B(a+j U)})^{-2}\right)\right) \\
& +O\left(R(n) T^{-3 / 2} n^{1 / 4}\right),
\end{aligned}
$$

where

$$
R(n)= \begin{cases}T^{-1 / 2} & \text { for } n<B(a+U) \\ 1 & \text { for } B(a+U) \leq n<B(a), \\ 0 & \text { for } n \geq B(a)\end{cases}
$$

Substitute (3.18) into (3.13). The first $O$-term in (3.18) then contributes

$$
\ll U^{-1} T^{3 / 4-\sigma} \sum_{j=0}^{1} \sum_{n=1}^{\infty} \sigma_{1-2 \sigma}(n) n^{\sigma-5 / 4} \min \left(1,(\sqrt{n}-\sqrt{B(a+j U)})^{-2}\right) .
$$

We claim that this is $\ll U^{-1} \ll T^{-1 / 4}$. In fact, split up the inner sum at $B / 2, B-\sqrt{B}, B+\sqrt{B}$ and $2 B$, where $B=B(a+j U) \asymp T$ (see (3.16)). The estimates of the first and the last subsums are easy. Since $\min (1,(\sqrt{n}-$ $\left.\sqrt{B})^{-2}\right) \leq 1$, it follows using $(2.1)$ that the middle subsum is $\ll U^{-1}$. The fourth subsum is decomposed further as

$$
\sum_{B+\sqrt{B} \leq n \leq 2 B}=\sum_{k} \sum_{B+2^{k} \sqrt{B} \leq n<B+2^{k+1} \sqrt{B}}
$$

and here we have $\min \left(1,(\sqrt{n}-\sqrt{B})^{-2}\right) \ll B(n-B)^{-2} \ll 2^{-2 k}$. Then we estimate each subsum by using (2.1), and sum up to obtain the estimate $O\left(U^{-1}\right)$. The second subsum can be treated similarly, so the claim is proved.

The second $O$-term in (3.18) contributes

$$
\ll T^{-\sigma} \sum_{n<B(a)} R(n) \sigma_{1-2 \sigma}(n) n^{\sigma-1}
$$


The terms with $n<B(a+U)$ contribute $\ll T^{-1 / 2}$. The other terms contribute

$$
\begin{aligned}
& \ll T^{-1} \sum_{B(a+U) \leq n<B(a)} \sigma_{1-2 \sigma}(n) \\
& \ll T^{-1}\left(B(a)-B(a+U)+T^{1 / 2}\right) \ll U T^{-1 / 2} \ll T^{-1 / 4},
\end{aligned}
$$

by $(2.1)$ and since $B^{\prime}(y)=-B(y)\left(T /(2 \pi)+y^{2} / 4\right)^{-1 / 2} \ll \sqrt{T}$ (see (3.16)).

On combining these results with (3.13) and (3.8) we get

$$
\begin{aligned}
G_{4}^{*}= & 2 i\left(\frac{T}{2 \pi}\right)^{1 / 2-\sigma} \sum_{n} \xi(T, n, a) \sigma_{1-2 \sigma}(n) n^{\sigma-1} \\
& \times\left(\log \frac{T}{2 \pi n}\right)^{-1} \cos \left(T \log \frac{T}{2 \pi n}-T+\frac{\pi}{4}\right) \\
& +O\left(T^{-1 / 4}+T^{1 / 4-\sigma}\right) .
\end{aligned}
$$

We have assumed that $a \asymp T^{1 / 2}$. Now let $V \asymp T^{1 / 2}$ and $a=V+v$, and denote by $w$ a differentiable function supported on the interval $[0,2 V]$ such that $w^{\prime}(v) \ll V^{-1}$ and $0 \leq w(v) \leq 1$ and

$$
\int w(v) d v=V \text {. }
$$

Define

$$
\widetilde{G}_{j}=V^{-1} \int w(v) G_{j}^{*} d v
$$

Then (3.10) yields

$$
G_{\sigma}(T)=\widetilde{G}_{1}-\widetilde{G}_{2}+\widetilde{G}_{3}-\widetilde{G}_{4} .
$$

By (3.9) and (3.5) we have

$$
\widetilde{G}_{1}=i \Sigma_{1, \sigma}(T)+O\left(T^{1 / 4-\sigma}\right)
$$

where

$$
\begin{aligned}
\Sigma_{1, \sigma}(T)= & \frac{1}{\sqrt{2}}\left(\frac{T}{2 \pi}\right)^{1 / 2-\sigma} \sum_{n} w_{1}(n)(-1)^{n} n^{\sigma-1} \sigma_{1-2 \sigma}(n) \\
& \times\left(\operatorname{arsinh} \sqrt{\frac{\pi n}{2 T}}\right)^{-1}\left(\frac{T}{2 \pi n}+\frac{1}{4}\right)^{-1 / 4} \cos (f(T, n)), \\
& w_{1}(n)=(V U)^{-1} \iint w(v) d u d v
\end{aligned}
$$

and the limits of the double integral are determined by the inequalities $0 \leq u \leq U$ and $n \leq(V+v+u)^{2}$. For our purpose it is sufficient to know that $0 \leq w_{1}(n) \leq 1$ and

$$
w_{1}(n)=\left\{\begin{array}{ll}
1 & \text { for } n \leq V^{2} \\
0 & \text { for } n \geq(3 V+U)^{2}
\end{array} .\right.
$$


Setting

$$
w_{2}(n, T)=V^{-1} \int w(v) \xi(T, n, V+v) d v
$$

we obtain, by (3.19),

$$
\widetilde{G}_{4}=i \Sigma_{2, \sigma}(T)+O\left(T^{-1 / 4}+T^{1 / 4-\sigma}\right),
$$

where

$$
\begin{aligned}
\Sigma_{2, \sigma}(T)= & 2\left(\frac{T}{2 \pi}\right)^{1 / 2-\sigma} \sum_{n} w_{2}(n, T) \sigma_{1-2 \sigma}(n) n^{\sigma-1} \\
& \times\left(\log \frac{T}{2 \pi n}\right)^{-1} \cos \left(T \log \frac{T}{2 \pi n}-T+\frac{\pi}{4}\right) .
\end{aligned}
$$

Writing $r=T /(2 \pi \sqrt{x})-\sqrt{x}$ we have

$$
w_{2}(x, T)=V^{-1} \int_{0}^{U} w(r-V-y) U^{-1} y d y+V^{-1} \int_{U}^{\infty} w(r-V-y) d y
$$

by (3.15). Since $w^{\prime}(v) \ll V^{-1}$, it follows that

$$
\frac{\partial}{\partial x} w_{2}(x, T)=\frac{\partial}{\partial r} w_{2}(x, T) \frac{\partial r}{\partial x} \ll V^{-1} T^{-1 / 2} \ll T^{-1}
$$

if $x \gg T$. But $x \gg T$ if $r<3 V+U$. And if $r \geq 3 V+U$ then $w_{2}(x, T)=1$ whence (3.27) holds trivially.

By (3.11) and (3.12),

$$
-\widetilde{G}_{2}+\widetilde{G}_{3}=-2 \pi i \zeta(2 \sigma-1)+O\left(T^{1 / 4-\sigma}+T^{-\sigma / 2+\varepsilon}+T^{\sigma-1}\right) .
$$

On combining (3.20), (3.21), (3.28) and (3.24) we find that

$$
G_{\sigma}(T)=i \Sigma_{1, \sigma}(T)-i \Sigma_{2, \sigma}(T)-2 \pi i \zeta(2 \sigma-1)+O\left(T^{-1 / 4}\right) .
$$

Together with (3.2) this gives finally

$$
E_{\sigma}(T)=\Sigma_{1, \sigma}(T)-\Sigma_{2, \sigma}(T)-2 \pi \zeta(2 \sigma-1)+O\left(T^{-1 / 4}\right),
$$

where $V \asymp T^{1 / 2}$ and $U \asymp T^{1 / 4}$.

Remark. For the proof of our theorem it would be sufficient to have $O\left(T^{-1 / 4+\varepsilon}\right)$ in (3.29). Averaging with respect to $a$ had no effect on the $O$ term in (3.29). The purpose of this averaging process is to provide $\Sigma_{2, \sigma}(T)$ with a weight function $w_{2}(n, T)$ of sufficiently slow decay. This makes it possible to save a logarithm in the estimation of $I_{22}$ in the next section.

4. The estimate for $F_{\sigma}(T)$. This section is based on Heath-Brown's [1] method (given also in [2]) supplied with ideas of Meurman [6] and Preissmann [10]. A new feature is the way we estimate $I_{22}$ (see below). HeathBrown's method alone would give Theorem 3 in [5] and adding the idea in [6] would yield $F_{\sigma}(T)=O(T \log T)$. 
Let $U=T^{1 / 4}$ and $V=\left(T^{1 / 2}-U\right) / 3$. By (3.29) and Schwarz's inequality we have

$$
\begin{aligned}
& \int_{T}^{2 T} E_{\sigma}(t)^{2} d t \\
= & I_{11}-2 I_{12}+I_{22}-4 \pi \zeta(2 \sigma-1) I_{1}+O\left(T^{1 / 4} I_{11}^{1 / 2}+T^{1 / 2} I_{22}^{1 / 2}+T\right),
\end{aligned}
$$

where

$$
I_{j k}=\int_{T}^{2 T} \Sigma_{j, \sigma}(t) \Sigma_{k, \sigma}(t) d t \quad \text { and } \quad I_{1}=\int_{T}^{2 T} \Sigma_{1, \sigma}(t) d t .
$$

Let $N=0$ or $N=T / A$, where $A$ is a large constant. Denote by $I_{11}(N)$ the integral which arises on replacing $\Sigma_{1, \sigma}(t)$ in $I_{11}$ by its subsum with $n>N$. Clearly we have $I_{11}(0)=I_{11}$.

Consider $I_{11}(N)$ and recall (3.22). Square out and integrate term-byterm. The non-diagonal terms give us

$$
\ll\left|\sum_{\substack{N<m \leq T \\ N<n \leq T \\ m \neq n}} a_{m} a_{n} \int_{T}^{2 T} k(t) e^{i \varphi(t)} d t\right|
$$

(see (3.23)), where

$$
\begin{gathered}
a_{n}=w_{1}(n)(-1)^{n} n^{\sigma-1} \sigma_{1-2 \sigma}(n) \\
k(t)=k_{m}(t) k_{n}(t) \\
k_{n}(t)=t^{1 / 2-\sigma}\left(\operatorname{arsinh} \sqrt{\frac{\pi n}{2 t}}\right)^{-1}\left(\frac{t}{2 \pi n}+\frac{1}{4}\right)^{-1 / 4}
\end{gathered}
$$

and

$$
\varphi(t)=f(t, m)-f(t, n) .
$$

(The sum with $f(t, m)+f(t, n)$ is easily estimated by using Lemma 2 of Heath-Brown [1].) The integral in (4.2) is by partial integration

$$
\left[-i \frac{k(t)}{\varphi^{\prime}(t)} e^{i \varphi(t)}\right]_{T}^{2 T}+i \int_{T}^{2 T} \frac{k(t)}{\varphi^{\prime}(t)}\left(\frac{k^{\prime}(t)}{k(t)}-\frac{\varphi^{\prime \prime}(t)}{\varphi^{\prime}(t)}\right) e^{i \varphi(t)} d t .
$$

We have $f^{\prime}(t, m)=2 \operatorname{arsinh} \sqrt{\pi m /(2 t)}$ (see $\left.(3.3)\right), k_{m}(t) \ll t^{3 / 4-\sigma} m^{-1 / 4}$, and for any fixed $m, \min _{n(\neq m)}\left|\varphi^{\prime}(t)\right| \gg(m t)^{-1 / 2}$. Hence the MontgomeryVaughan inequality (see [7]) gives us

$$
\sum_{m \neq n} a_{m} a_{n} \frac{k(t)}{\varphi^{\prime}(t)} e^{i \varphi(t)} \ll \sum_{N<n \leq T} a_{n}^{2} k_{n}(t)^{2}(n t)^{1 / 2} \ll T
$$


for $t=T$ and $t=2 T$ (see also Corollary of Preissmann [10]). Hence and by (4.3) we see that the expression in (4.2) is

$$
\ll T+\int_{T}^{2 T}\left|\sum_{m \neq n} a_{m} a_{n} \frac{k(t)}{\varphi^{\prime}(t)}\left(\frac{k^{\prime}(t)}{k(t)}-\frac{\varphi^{\prime \prime}(t)}{\varphi^{\prime}(t)}\right) e^{i \varphi(t)}\right| d t .
$$

Since $f^{\prime \prime}(t, m)=-t^{-1} \tanh \left(f^{\prime}(t, m) / 2\right),(\tanh x)^{\prime}=(\cosh x)^{-2}$ and $(\tanh x)^{\prime \prime}$ $\ll 1$, it follows that

$$
\varphi^{\prime \prime}(t)=-\frac{1}{2 t} \varphi^{\prime}(t)\left(\cosh \left(\frac{1}{2} f^{\prime}(t, m)\right)\right)^{-2}+O\left(\frac{\varphi^{\prime}(t)^{2}}{t}\right) .
$$

The $O$-term here contributes trivially $\ll T$. Therefore $\varphi^{\prime \prime}(t) / \varphi^{\prime}(t)$ may be replaced by a function $g(t)$ such that $g(t)=O(1 / t)$ and $g(t)$ depends on only one of the variables $m$ and $n$. A simple calculation shows that $k^{\prime}(t) / k(t)$ is a sum of functions sharing these properties. Now, after using the Montgomery-Vaughan inequality, we conclude that the expression in (4.4) is $O(T)$.

Following Heath-Brown's [1] argument and using (3.23), we see that the diagonal terms of $I_{11}(N)$ yield

$$
\frac{2(2 \pi)^{2 \sigma-3 / 2}}{5-4 \sigma} \sum_{n>N} \sigma_{1-2 \sigma}(n)^{2} n^{2 \sigma-5 / 2}\left((2 T)^{5 / 2-2 \sigma}-T^{5 / 2-2 \sigma}\right)+O(T) .
$$

For $N=T / A$ this is $O(T)$ and for $N=0$ we may determine the value of the series by Ramanujan's identity (see formula (1.3.3) in [12]). Altogether we have now

$$
I_{11}=c_{0}\left((2 T)^{5 / 2-2 \sigma}-T^{5 / 2-2 \sigma}\right)+O(T),
$$

where $c_{0}$ is given in Section 1 , and

$$
I_{11}(T / A) \ll T .
$$

Consider $I_{22}$. By (3.25) we have

$$
\Sigma_{2, \sigma}(t) \ll T^{1 / 2-\sigma}\left|\sum_{n} w_{2}(n, t) \sigma_{1-2 \sigma}(n)\left(\log \frac{t}{2 \pi n}\right)^{-1} n^{\sigma-1+i t}\right| .
$$

Let us remove $w_{2}(n, t)(\log (t /(2 \pi n)))^{-1}$ by partial summation. By (3.26) and (3.17) we have $w_{2}(x, t)=0$ if $x \geq B(V, t)$. If $x<B(V, t)$, then $\log (t /(2 \pi x)) \gg 1$, by $(3.17)$. Noting also that $B(V, t) \leq T$ and $w_{2}(x, t) \ll 1$ and using (3.27) we obtain

$$
\Sigma_{2, \sigma}(t) \ll T^{1 / 2-\sigma} \int_{1}^{T} \frac{1}{x \log ^{2}(2 T / x)}\left|\sum_{n \leq x} \sigma_{1-2 \sigma}(n) n^{\sigma-1+i t}\right| d x .
$$

Hence, using Schwarz's inequality and changing the order of the integrations, 
we obtain

$$
I_{22} \ll T^{1-2 \sigma} \int_{1}^{T} \frac{1}{x} \int_{T}^{2 T}\left|\sum_{n \leq x} \sigma_{1-2 \sigma}(n) n^{\sigma-1+i t}\right|^{2} d t d x .
$$

Here we use the mean value theorem for Dirichlet polynomials (see Theorem 5.2 in [2]) to obtain

$$
I_{22} \ll T \text {. }
$$

To estimate $I_{12}$ we follow closely the argument in [6] and make use of (4.6) and (4.7). Here it is important that $A$ is large. We obtain

$$
I_{12} \ll T \text {. }
$$

An integration term-by-term gives easily

$$
I_{1} \ll T^{5 / 4-\sigma} .
$$

Combining this with (4.1), (4.5), (4.7) and (4.8) yields

$$
\int_{T}^{2 T} E_{\sigma}(t)^{2} d t=c_{0}\left((2 T)^{5 / 2-2 \sigma}-T^{5 / 2-2 \sigma}\right)+O(T) .
$$

Our theorem follows from this immediately.

Appendix. Here we prove that $c_{3}=0$ for $1 / 2<\sigma<1$. It is enough to show that

$$
\int_{0}^{\infty}\left(\frac{1}{\pi} \operatorname{ch}(\pi t)|\Gamma(1-\sigma+i t)|^{2}-t^{1-2 \sigma}\right) d t=0
$$

Define

$$
H(w)=\int_{0}^{\infty}\left(\frac{|\Gamma(1-\sigma+i t)|^{2}}{\Gamma(1 / 2+w+i t) \Gamma(1 / 2+w-i t)}-e^{-1 / t} t^{1-2 \sigma-2 w}\right) d t
$$

and let $K$ be any compact subset of the half-plane $\operatorname{Re}(w)>1 / 2-\sigma$. For any $w \in K$ and any $t \geq \max _{z \in K}|\operatorname{Im}(z)|+1$ we can by Stirling's formula show that

$$
\frac{|\Gamma(1-\sigma+i t)|^{2}}{\Gamma(1 / 2+w+i t) \Gamma(1 / 2+w-i t)}=t^{1-2 \sigma-2 w}\left(1+O\left(t^{-1}\right)\right) .
$$

It follows that the integral $H(w)$ is uniformly convergent in $K$. Hence $H(w)$ is holomorphic in $\operatorname{Re}(w)>1 / 2-\sigma$. 
Now, let $w$ be real, satisfying $w>3 / 2-\sigma$. Then

$$
\begin{aligned}
\int_{0}^{\infty}\left|\frac{\Gamma(1-\sigma+i t)}{\Gamma(1 / 2+w+i t)}\right|^{2} d t & =\frac{1}{2 i} \int_{-i \infty}^{i \infty}\left|\frac{\Gamma(1-\sigma+z)}{\Gamma(1 / 2+w+z)}\right|^{2} d z \\
& =\frac{\pi \Gamma(2-2 \sigma) \Gamma(2 w+2 \sigma-2)}{\Gamma(w+\sigma-1 / 2)^{2} \Gamma(2 w)},
\end{aligned}
$$

where the last equality follows from (7.8.4) of Titchmarsh [11]. Titchmarsh stated (7.8.4) under the conditions $-\alpha<k,-\beta<k, \gamma>k$ and $\delta>k$, but it seems that the further conditions $\beta>\alpha$ and $\delta>\gamma+1$ (or, instead, the conditions $\beta>\alpha+1$ and $\delta>\gamma$ ) are necessary, because (7.8.4) is verified by using Theorem 42 in [11]. Therefore, in our case we assume $w>3 / 2-\sigma$. We now obtain

$$
H(w)=\frac{\pi \Gamma(2-2 \sigma) \Gamma(2 w+2 \sigma-2)}{\Gamma(w+\sigma-1 / 2)^{2} \Gamma(2 w)}-\Gamma(2 \sigma+2 w-2)
$$

for $w>3 / 2-\sigma$, and by analytic continuation we have $H(0)=-\Gamma(2 \sigma-2)$. Our claim follows from this fact and the formulas

$$
\int_{0}^{\infty} t^{1-2 \sigma}\left(e^{-1 / t}-1\right) d t=\Gamma(2 \sigma-2)
$$

and $|\Gamma(1 / 2+i t)|^{2}=\pi / \operatorname{ch}(\pi t)$.

N ot e. This article was completed already in 1991, and was submitted to J. Number Theory in early 1992, but was withdrawn in early 1994, because J. Number Theory finally replied "We have no record of your paper". Meanwhile a subsequent paper The mean square of the Riemann zeta-function in the critical strip III was published in Acta Arith. 64 (1993), 357-382. In the references of that paper, the publication data of the present paper and of [13] are to be interchanged.

\section{References}

[1] D. R. Heath-Brown, The mean value theorem for the Riemann zeta-function, Mathematika 25 (1978), 177-184.

[2] A. Ivić, The Riemann Zeta-Function, Wiley, New York, 1985.

[3] -, Lectures on Mean Values of the Riemann Zeta-Function, Lectures on Math. and Physics 82, Tata Inst. Fund. Res., Springer, Bombay, 1991.

[4] I. Kiuchi, On an exponential sum involving the arithmetic function $\sigma_{a}(n)$, Math. J. Okayama Univ. 29 (1987), 193-205.

[5] K. Matsumoto, The mean square of the Riemann zeta-function in the critical strip, Japan. J. Math. 15 (1989), 1-13.

[6] T. Meurman, On the mean square of the Riemann zeta-function, Quart. J. Math. Oxford (2) 38 (1987), 337-343.

[7] H. Montgomery and R. C. Vaughan, Hilbert's inequality, J. London Math. Soc. (2) 8 (1974), 73-82. 
[8] Y. Motohashi, A note on the mean value of the zeta and L-functions $I V$, Proc. Japan Acad. Ser. A 62 (1986), 311-313.

[9] -, Lectures on the Riemann-Siegel formula, Ulam Seminar, Department of Math., University of Colorado, Boulder, 1987.

[10] E. Preissmann, Sur la moyenne de la fonction zêta, in: Analytic Number Theory and Related Topics, K. Nagasaka (ed.), World Scientific, 1993, 119-125.

[11] E. C. Titchmarsh, Introduction to the Theory of Fourier Integrals, Oxford Univ. Press, London, 1937.

[12] - , The Theory of the Riemann Zeta-Function, Oxford Univ. Press, London, 1951.

DEPARTMENT OF MATHEMATICS

FACULTY OF EDUCATION

IWATE UNIVERSITY

MORIOKA 020, JAPAN
DEPARTMENT OF MATHEMATICS UNIVERSITY OF TURKU SF-20500 TURKU, FINLAND 